\title{
Orbit Determination of Space Debris: Admissible Regions
}

\author{
Tommei G. ${ }^{1}$, Milani A. ${ }^{1}$ and Rossi A. ${ }^{2}$ \\ ${ }^{1}$ Department of Mathematics, University of Pisa \\ Largo B. Pontecorvo 5, 56127 Pisa, Italy \\ e-mail: tommei@dm.unipi.it, milani@dm.unipi.it \\ 2 ISTI/CNR, Research Area of Pisa \\ Via Moruzzi, 1, 56124 Pisa - Italy \\ e-mail: alessandro.rossi@isti.cnr.it
}

\begin{abstract}
The main problem in the orbit determination of the space debris population orbiting our planet is identifying which separate sets of data belong to the same physical object. The observations of a given object during a passage above an observing station are collectively called a Too Short Arc (TSA): data from a TSA cannot allow for a complete determination of an orbit. Therefore we have to solve first the identification problem, finding two or more TSAs belonging to the same physical object and an orbit fitting all the observations. This problem is well known for the determination of orbits of asteroids: we shall show how to apply the methods developed for preliminary orbit determination of heliocentric objects to geocentric objects. We shall focus on the definition of an admissible region for space debris, both in the case of optical observations and radar observations; then we shall outline a strategy to perform a full orbit determination.
\end{abstract}

Keywords: space debris, orbit determination, admissible region 


\section{Introduction}

The near-Earth space is filled by more than 300000 artificial debris particles with diameter larger than $1 \mathrm{~cm}$ [Rossi, 2005a]. This population is similar to the asteroidal one because its long term evolution is affected by high-velocity mutual collisions. The space where the debris is placed can be divided into three main regions: the Low Earth Orbit (LEO), below about $2000 \mathrm{~km}$, the Medium Earth Orbit (MEO), between $2000 \mathrm{~km}$ and about $36000 \mathrm{~km}$, and the Geosynchronous Earth Orbit (GEO) at about $36000 \mathrm{~km}$ of altitude.

The orbits of all the unclassified spacecraft and space debris are collected by the United States Air Force Space Command (AFSPC) in the TwoLine Element (TLE) catalog and are updated thanks to the observations performed by a network of 25 sensors, both radars and optical, called the Space Surveillance Network (SSN). Radar is mainly used to track objects in Low and Medium Earth Orbit, while, above several thousand $\mathrm{km}$ of altitude, the radar power is not sufficient to monitor the small space debris and the SSN uses optical sensors. Tracking and data processing is now automated and about 80000 observations are processed daily by the SSN. In order to characterize the GEO environment the European Space Agency (ESA) has installed a $1 \mathrm{~m}$ Schmidt telescope on the Canary Islands with a limiting detection size of about $15 \mathrm{~cm}$. Note that the space debris is also observed by astronomers worldwide. See, e.g., [Rossi, 2005a] and [Rossi, 2005b] for further details on observation for space debris and on population models.

In this paper we shall outline the theoretical concepts that will constitute the basis of an orbit determination program of space debris. The main problem to be solved to compute the orbits of the observed space debris is the identification (also called correlation) of two or more set of observational data. A piece of space debris passing above an observing station and observable for some short time is called a pass (for a geosynchronous satellite, a pass can be defined by the duration of the observing time during one night). The observations of a given object during a pass are called a Too Short Arc (TSA), also called a tracklet or uncorrelated track. Data from a TSA are believed to belong to the same object because they can be joined by some smooth curve (in most cases, simply a straight line, or a great circle); as an example, if the image moves with the fixed stars, the debris produces a trail of which the two extremes are measured.

The data from one TSA cannot allow for the determination of an orbit: e.g., if only two angular observations are available, as in the case of a trail, there are 4 equations in 6 unknown orbital elements. Thus it is not possible to solve the orbit determination problem without solving first 
the identification problem, finding two or more TSA belonging to the same physical object and an orbit fitting all the observations (linkage between two or more TSAs). The above discussion applies to optical observations, but it is possible to formulate a similar problem for radar data.

This problem has a very dangerous computational complexity, increasing at least like the square of the number of TSA, thus increasing very sharply with the size of the observable population, that is with the limiting magnitude of the survey. If the problem of identification is not solved the data do not provide orbits for any object. If the identification problem is solved in an incomplete way then a significant fraction of the observational data remain locked in the TSA and have no practical use, apart from an approximate estimate of the population size.

This problem is well known for the determination of heliocentric orbits (e.g. asteroids and comets) and has been studied intensively in recent years because of the development of high-technology wide-field surveys to discover, among other astronomical objects, most asteroids and comets down to small sizes. The solution proposed by [Milani et al., 2004] and [Milani and Knežević, 2005] uses the concept of attributable, a four dimensional quantity defined by two or more observations and synthesizing the useful information from a TSA. From the attributable we can compute an admissible region of orbits bound to the solar system, which is a compact subset of the range, range rate half plane. The admissible region can be sampled, for example by an optimal triangulation, generating Virtual Asteroids (VAs), see [Milani and Chesley, 2000], which can be used as alternate preliminary orbits. The latter can be used to start a recursive procedure of identification and orbit determination, which has been tested on simulations of the next generation surveys [Milani et al., 2005b]. The simulations have shown that the problem can be solved for a number of TSAs of the order of 1 million collected in each night of survey.

The same technique can be used for space debris. We shall modify the definition of admissible region taking into account the orbits of the Earth's satellites. We shall develop the theory for optical observations (Section 2), used for debris in high orbits, and for radar observations (Section 3), used for objects in Low and Medium orbits. In Subsection 2.1 we shall explain how to obtain a finite number of orbits sampling the admissible region with an optimal triangulation, while in Subsection 3.1 we shall explain a new type of sampling useful when the region is an ellipse. In Section 4 we shall present the strategy to perform a full orbit determination, involving also the Line Of Variations (LOV) tool. The LOV can be described as a string, that is a one-dimensional segment of a curved line, in the initial conditions 
space. The general idea is that a segment of this line is a kind of spine of the region, in the orbital elements space, containing the candidate objects. See [Milani et al., 2005a] for the rigorous definition of LOV and its applications.

The optimization of this procedure needs to be performed by testing on a large enough data set, but it is clear that the current sets of the scientific campaigns of debris optical observations are not as big as the ones of the next generation asteroid surveys (simply because smaller telescopes and cameras are used). On the other hand the routine tracking and data processing performed by the SSN to maintain the TLE Catalog is automated and about 80000 observations are processed daily.

A future work will be the development of the software to process real data.

\section{Admissible region from optical observations}

In this section we shall define analytically the admissible region for a space debris object observed by a ground-based optical sensor. The admissible region replaces the conventional confidence region as defined in the classical orbit determination procedure. The main requirement is that the geocentric energy of the object is negative, that is the object is a satellite of the Earth. Let $P$ be the geocentric position of an orbiting space debris $\mathcal{D}$ observed at a time $t$. Then

$$
P=P_{O}+P_{\mathcal{D}}
$$

where $P_{O}$ is the geocentric position of the observer $\mathrm{O}$ and $P_{\mathcal{D}}=\rho \widehat{R}$ is the vector in the observation direction.

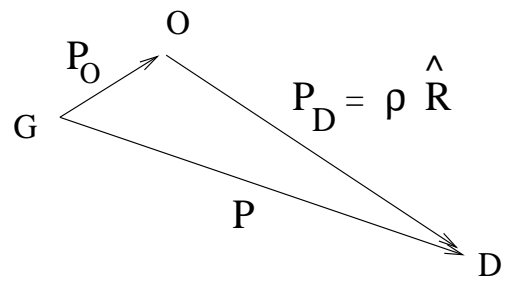

Figure 1: The space debris $\mathcal{D}$ is observed by the point $\mathrm{O}$ on the surface of the Earth. The geocenter is denoted by $\mathrm{G}$.

Let

$$
(\rho, \alpha, \delta) \in \mathbb{R}^{+} \times[-\pi, \pi) \times(-\pi / 2, \pi / 2)
$$

be the spherical polar coordinates defining the vector $P_{\mathcal{D}}$. 
DeFinition 1. An optical attributable is a vector

$$
A_{\text {opt }}=(\alpha, \delta, \dot{\alpha}, \dot{\delta}) \in[-\pi, \pi) \times(-\pi / 2, \pi / 2) \times \mathbb{R}^{2},
$$

observed at a time $t$.

As reference system for the polar coordinates we normally use an equatorial one (e.g., J2000), that is $\alpha$ is the right ascension and $\delta$ the declination; note that it is possible to change the reference system without modifying the equations in the paper.

Since the range $\rho$ and the range rate $\dot{\rho}$ are left undetermined by the attributable, following [Milani et al., 2004] we shall derive conditions on $(\rho, \dot{\rho})$ under the hypothesis that the object $\mathcal{D}$ is a satellite of the Earth. The quantities used are the following:

- Geocentric two-body energy per unit mass of the object

$$
\mathcal{E}_{E}(\rho, \dot{\rho})=\frac{1}{2}\|\dot{P}\|^{2}-\frac{\mu_{E}}{\|P\|},
$$

where $\mu_{E}=G m_{E}$ and $m_{E}$ is the Earth mass.

- Lower bound for the space debris distance from the position of the observer on the Earth

$$
\rho_{M I N}=2 r_{E} \simeq 12756 \mathrm{~km},
$$

where $r_{E}$ is the value of the Earth radius. We use this bound because we are interested in space debris in high orbits; the objects in lower orbits are usually observed by radar and the admissible region changes (see Section 3).

- Upper bound for the space debris distance from the position of the observer on the Earth

$$
\rho_{M A X}=20 r_{E} \simeq 127560 \mathrm{~km} .
$$

Note that we have set the values of $\rho_{M I N}$ and $\rho_{M A X}$ "a priori" and that we could vary them during the analysis of real data. The current choice of $\rho_{M I N}$ is driven by the fact that the region between $\approx 2000 \mathrm{~km}$ and $\rho_{M I N}$ shows an extremely low density of objects and is therefore not of interest for any observation campaign. Now let us write the conditions on $(\rho, \dot{\rho})$ explicitly: 
(A) $\mathcal{C}_{1}=\left\{(\rho, \dot{\rho}): \mathcal{E}_{E}<0\right\}$ (D is a satellite of the Earth);

(B) $\mathcal{C}_{2}=\left\{(\rho, \dot{\rho}): \rho_{M I N}<\rho<\rho_{M A X}\right\}$ (the distance of the object by the observer is in the interval $\left.\left(\rho_{M I N}, \rho_{M A X}\right)\right)$.

Another condition should be taken into account, that is, that $\mathcal{D}$ belongs to the Solar System (the heliocentric energy $\mathcal{E}_{S}$ of the object should be negative). However, [Milani et al., 2004] have proved that if the object is controlled by the Earth $\left(r \leq r_{S I}\right.$, where $r$ is the geocentric distance of the object and $r_{S I}$ the radius of the sphere of influence of the Earth) then a non-positive geocentric energy implies a non-positive heliocentric energy ${ }^{1}$. By adding the upper bound for the distance $\rho_{M A X}$ and the Earth radius $r_{E}$, we obtain a quantity that is less than $r_{S I}$, therefore $r<r_{S I}$ and we do not care about the condition on the heliocentric energy.

Definition 2. Given an attributable $A_{\text {opt }}$, we define as admissible region for a space debris $\mathcal{D}$ the set

$$
\mathcal{C}=\mathcal{C}_{1} \cap \mathcal{C}_{2}
$$

Condition (B) bounds only $\rho$, therefore the region $\mathcal{C}_{2}$ appears as an infinite stripe in the $(\rho, \dot{\rho})$ plane. Now we look for a geometrical and analytical description of the region defined by the condition (A) on the geocentric energy. The geocentric position of $\mathcal{D}$ is given by (1). Using polar coordinates (2), the geocentric velocity $\dot{P}$ of $\mathcal{D}$ is

$$
\dot{P}=\dot{P}_{O}+\dot{\rho} \widehat{R}+\rho \dot{\alpha} \widehat{R}_{\alpha}+\rho \dot{\delta} \widehat{R}_{\delta},
$$

where $\dot{P}_{O}$ is the geocentric velocity of the observer and

$$
\widehat{R}_{\alpha}=\frac{\partial \widehat{R}}{\partial \alpha}, \quad \widehat{R}_{\delta}=\frac{\partial \widehat{R}}{\partial \delta} .
$$

Explicitly in coordinates

$$
\begin{aligned}
\widehat{R} & =(\cos \alpha \cos \delta, \sin \alpha \cos \delta, \sin \delta) \\
\widehat{R}_{\alpha} & =(-\sin \alpha \cos \delta, \cos \alpha \cos \delta, 0) \\
\widehat{R}_{\delta} & =(-\cos \alpha \sin \delta,-\sin \alpha \sin \delta, \cos \delta) .
\end{aligned}
$$

Note that the vectors $\widehat{R}, \widehat{R}_{\alpha}, \widehat{R}_{\delta}$ define an orthogonal basis for $\mathbb{R}^{3}$; this basis is not orthonormal because $\left\|\widehat{R}_{\alpha}\right\|=\cos \delta$.

\footnotetext{
${ }^{1}$ This assumption is valid only for particular values of the mass, radius and orbital parameters of the planet on which the observer is located
} 
In order to compute the geocentric energy (4) we use the geocentric position and velocity in polar coordinates:

$$
\begin{aligned}
\|P\|^{2}= & \rho^{2}+2 \rho\left\langle P_{O}, \widehat{R}\right\rangle+\left\|P_{O}\right\|^{2}, \\
\|\dot{P}\|^{2}= & \dot{\rho}^{2}+2 \dot{\rho}\left\langle\dot{P}_{O}, \widehat{R}\right\rangle+\rho^{2}\left(\dot{\alpha}^{2} \cos ^{2} \delta+\dot{\delta}^{2}\right)+ \\
& 2 \rho\left(\dot{\alpha}\left\langle\dot{P}_{O}, \widehat{R}_{\alpha}\right\rangle+\dot{\delta}\left\langle\dot{P}_{O}, \widehat{R}_{\delta}\right\rangle\right)+\left\|\dot{P}_{O}\right\|^{2} .
\end{aligned}
$$

Introducing the notation

$$
\begin{array}{rlrl}
w_{0} & =\left\|P_{O}\right\|^{2} & w_{3} & =\dot{\alpha} w_{3, \alpha}+\dot{\delta} w_{3, \delta} \\
w_{1} & =2\left\langle\dot{P}_{O}, \widehat{R}\right\rangle & w_{4}=\left\|\dot{P}_{O}\right\|^{2} \\
w_{2} & =\dot{\alpha}^{2} \cos ^{2} \delta+\dot{\delta}^{2} & w_{5} & =2\left\langle P_{O}, \widehat{R}\right\rangle,
\end{array}
$$

where

$$
\begin{aligned}
& w_{3, \alpha}=2\left\langle\dot{P}_{O}, \widehat{R}_{\alpha}\right\rangle \\
& w_{3, \delta}=2\left\langle\dot{P}_{O}, \widehat{R}_{\delta}\right\rangle
\end{aligned}
$$

we have

$$
\begin{aligned}
& \|P\|^{2}=\rho^{2}+w_{5} \rho+w_{0}, \\
& \|\dot{P}\|^{2}=\dot{\rho}^{2}+w_{1} \dot{\rho}+w_{2} \rho^{2}+w_{3} \rho+w_{4} .
\end{aligned}
$$

By substituting in eq. (4), condition (A) becomes

$$
2 \mathcal{E}_{E}(\rho, \dot{\rho})=\dot{\rho}^{2}+w_{1} \dot{\rho}+T(\rho)-\frac{2 \mu_{E}}{\sqrt{S(\rho)}}<0,
$$

where

$$
\begin{aligned}
& S(\rho)=\rho^{2}+w_{5} \rho+w_{0}, \\
& T(\rho)=w_{2} \rho^{2}+w_{3} \rho+w_{4} .
\end{aligned}
$$

In order to obtain real solutions for $\dot{\rho}$ the discriminant of $2 \mathcal{E}_{E}$ (polynomial of degree 2 in $\dot{\rho}$ ) must be non-negative:

$$
\Delta_{E}=\frac{w_{1}^{2}}{4}-T(\rho)+\frac{2 \mu_{E}}{\sqrt{S(\rho)}} \geq 0 .
$$

This observation results in the following condition on $\rho$ :

$$
\frac{2 \mu_{E}}{\sqrt{S(\rho)}} \geq Q(\rho)
$$


where

$$
Q(\rho)=w_{2} \rho^{2}+w_{3} \rho+\gamma
$$

with

$$
\gamma=w_{4}-\frac{w_{1}^{2}}{4}
$$

Note that the polynomial $Q(\rho)$ is non-negative: in fact

$$
Q(\rho)=-\Delta_{K(\rho, \dot{\rho})},
$$

where $\Delta_{K(\rho, \dot{\rho})}$ is the discriminant of the 2-degree polynomial representing the kinetic energy $K$ as a function of $\dot{\rho}$. Since $K$ is non-negative its discriminant is non-positive and then $Q(\rho)$ is non-negative.

Condition (6) can be rewritten as an inequality involving a polynomial $V(\rho)$ of degree 6 :

$$
V(\rho):=Q^{2}(\rho) S(\rho) \leq 4 \mu_{E}^{2} .
$$

Studying the polynomial $V(\rho)$ and its roots, as done by [Milani et al., 2004], the conclusion is that the region $\mathcal{C}_{1}$, defined by condition $(\mathrm{A})$, can admit more than one connected components, but it has at most two. In the case of orbit determination of heliocentric bodies there are examples of admissible regions with two components, but to investigate if this happens also for space debris we need to analyze real or simulated data.

The qualitative structure of the confidence region is shown in the next figures. Figure 2 shows the case with one connected component: on the left $\rho_{M A X}$ is less than the range of the curve $\mathcal{E}_{E}=0$ corresponding to $\dot{\rho}=0$, while on the right it is greater. Figure 3 shows the case with two connected components: on the left $\rho_{M A X}$ is less then the minimum range corresponding to the second connected component of the curve $\mathcal{E}_{E}=0$, while on the right it is greater.

In order to exclude orbits of "just-launched" objects, we should add a third condition on the pericenter $q=a(1-e)$ ( $a$ is the semimajor axis and $e$ the eccentricity), that is $q>r_{E}+h$, where $h$ could be the height of the atmosphere. But to compute analytically the corresponding curve in the $(\rho, \dot{\rho})$ plane is extremely complicated. However we have thought that it is possible to make a restriction on the semimajor axis that corresponds to fixing a lower bound for the energy, by replacing condition (A) with

$\left(\mathrm{A}^{\prime}\right) \mathcal{C}_{1}=\left\{(\rho, \dot{\rho}): \mathcal{E}_{E}^{\min }<\mathcal{E}_{E}<0\right\}$ ( $\mathcal{D}$ is a satellite of the Earth and its semimajor axis is greater than a fixed quantity).

Figure 4 shows the resulting admissible region (for simplicity we show only the case with one connected component). 

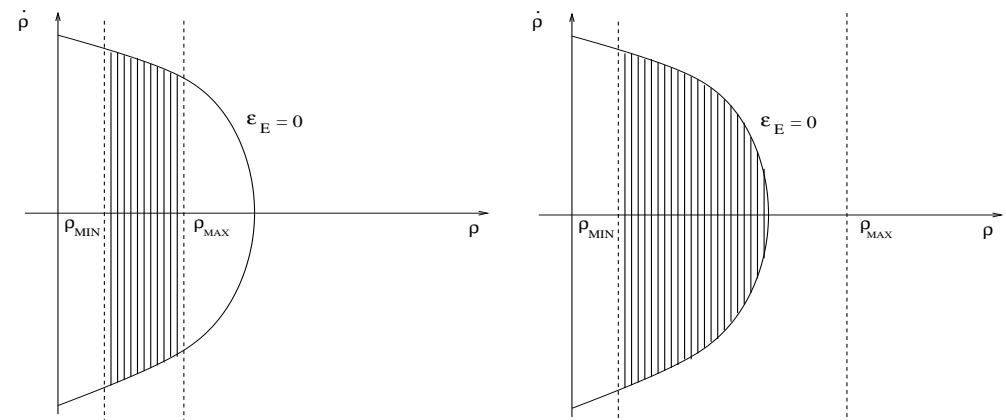

Figure 2: Admissible region for a space debris $\mathcal{D}$ from optical data (one connected component): $\mathcal{E}_{E}=0$ is the curve of zero geocentric energy, $\rho_{M I N}$ and $\rho_{M A X}$ are the lower and the upper limit for the distance of $\mathcal{D}$ from the observer.
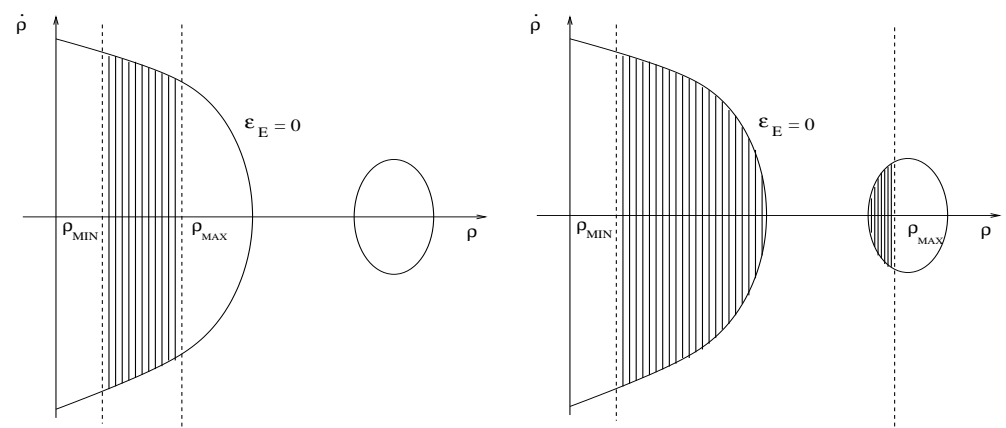

Figure 3: Admissible region for space debris $\mathcal{D}$ from optical data (two connected components): $\mathcal{E}_{E}=0$ is the curve of zero geocentric energy, $\rho_{M I N}$ and $\rho_{M A X}$ are the lower and the upper limit for the distance of $\mathcal{D}$ from the observer.

\subsection{Sampling of the admissible region with triangulation}

Given a very short arc of observations and the corresponding optical attributable, $A_{\text {opt }}=(\alpha, \delta, \dot{\alpha}, \dot{\delta})$, at some epoch (for example the mean observation time), a least square solution for the orbital elements, with its normal and covariance matrix, (see [Milani et al., 2005a] for more details on orbit determination), is in general not computable.

If we assume that the object is a satellite of the Earth we can limit the uncertainty to the appropriate admissible region in the $(\rho, \dot{\rho})$ plane. The admissible region is a compact subset of the plane in which it is defined: however, this is still an infinite set, thus there are infinite possible orbits 


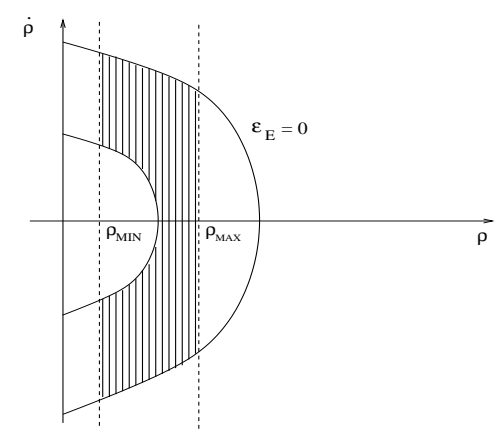

Figure 4: Admissible region for a space debris $\mathcal{D}$ taking into account the condition $\left(\mathrm{A}^{\prime}\right)$ instead of (A).

and we need to find efficient ways to sample them with a finite number of points obtaining a set of Virtual Debris (VD). They are the equivalent of VAs in the case of heliocentric orbits: they share the reality of the object in the sense that the orbit represented by one of them is a good approximation of the orbit of the real object, but we do not know which one.

This requires to sample the admissible region $\mathcal{C}$ with a finite number of points. The most natural and geometrically significant way to sample a two dimensional region is a triangulation, with nodes and sides joining them. Since we know the analytical expression of the boundary of the admissible region, the Delaunay triangulation, which has a number of optimal properties, could be a useful choice: in fact there is an efficient algorithm to compute it, starting from a finite sampling of the boundary [Milani et al., 2004]. Then the nodes are selected as points $\left(\rho_{i}, \dot{\rho}_{i}\right), i=1, N$ sampling the admissible region, with the sides and the triangles providing an additional geometric structure. In this way we have obtained a discrete set of orbits to use in an identification process as described in Section 4.

\section{Admissible region from radar data}

When a radar observation is performed we assume that the quantities measured are the range, the range rate, and also the position on the celestial sphere (all with their own uncertainty), expressed by two angular coordinates, such as right ascension $\alpha$ and declination $\delta$ [Mehrholz et al., 2002]. Then the time derivatives of these angular coordinates, $\dot{\alpha}$ and $\dot{\delta}$, are undetermined: therefore the concept of attributable must be modified and the admissible region computed in the $(\dot{\alpha}, \dot{\delta})$ plane. 
DEFINITION 3. A radar attributable is a vector

$$
A_{\text {rad }}=(\alpha, \delta, \rho, \dot{\rho}) \in[-\pi, \pi) \times(-\pi / 2, \pi / 2) \times \mathbb{R}^{+} \times \mathbb{R},
$$

observed at a time $t$.

Since the distance of the object is measured by the radar observation the definition of admissible region in this case is the following.

Definition 4. Given an attributable $A_{\text {rad }}$, we define as admissible region for a space debris $\mathcal{D}$ the set

$$
\mathcal{C}=\mathcal{C}_{1}
$$

In order to compute analytically the admissible region we have to express the geocentric energy (and therefore condition (5)) as function of $\dot{\alpha}$ and $\dot{\delta}$, that are the quantities undetermined:

$$
2 \mathcal{E}_{E}=\dot{\rho}^{2}+w_{1} \dot{\rho}+w_{2} \rho^{2}+w_{3} \rho+w_{4}-\frac{2 \mu_{E}}{\sqrt{S(\rho)}} .
$$

Only the coefficients $w_{2}$ (the proper motion) and $w_{3}$ depend on $\dot{\alpha}$ and $\dot{\delta}$, thus expanding the previous expression we have the following polynomial of the second order in $\dot{\alpha}$ and $\dot{\delta}$

$$
F(\dot{\alpha}, \dot{\delta})=z_{11} \dot{\alpha}^{2}+2 z_{12} \dot{\alpha} \dot{\delta}+z_{22} \dot{\delta}^{2}+2 z_{13} \dot{\alpha}+2 z_{23} \dot{\delta}+z_{33},
$$

with a $3 \times 3$ coefficient matrix

$$
Z=\left(\begin{array}{lll}
z_{11} & z_{12} & z_{13} \\
z_{21} & z_{22} & z_{23} \\
z_{31} & z_{32} & z_{33}
\end{array}\right)
$$

where

$$
\begin{array}{llll}
z_{11} & =\rho^{2} \cos ^{2} \delta & z_{13}=z_{31} & =\rho\left\langle\dot{P}_{O}, \widehat{R}_{\alpha}\right\rangle, \\
z_{12}=z_{21} & =0 & z_{23}=z_{32} & =\rho\left\langle\dot{P}_{O}, \widehat{R}_{\delta}\right\rangle, \\
z_{22} & =\rho^{2} & z_{33} & =\dot{\rho}^{2}+w_{1} \dot{\rho}+w_{4}-\frac{2 \mu_{E}}{\sqrt{S(\rho)}}
\end{array}
$$

The border of the admissible region in the $(\dot{\alpha}, \dot{\delta})$ plane is then given by

$$
F(\dot{\alpha}, \dot{\delta})=0,
$$

expressed in matricial form by

$$
k Z k^{T}=0,
$$


where $k$ is the 3 -dimensional row vector $(\dot{\alpha}, \dot{\delta}, 1)$.

Equation (10) represents a conic section in the $(\dot{\alpha}, \dot{\delta})$ plane. Let us examine the invariants of the polynomial $F(\dot{\alpha}, \dot{\delta})$ to investigate the type of conics.

The linear invariant is the trace of the $2 \times 2$ matrix of the coefficients of the second order:

$$
\operatorname{tr} Z_{33}=\operatorname{tr}\left(\begin{array}{ll}
z_{11} & z_{12} \\
z_{21} & z_{22}
\end{array}\right)=z_{11}+z_{22}=\rho^{2}\left(\cos ^{2} \delta+1\right)>0 .
$$

The quadratic invariant is the determinant of the $2 \times 2$ matrix of the coefficients of the second order:

$$
\operatorname{det} Z_{33}=\operatorname{det}\left(\begin{array}{ll}
z_{11} & z_{12} \\
z_{21} & z_{22}
\end{array}\right)=z_{11} z_{22}=\rho^{4} \cos ^{2} \delta>0 .
$$

The cubic invariant is the determinant of the matrix $Z$ :

$$
\operatorname{det} Z=\operatorname{det}\left(\begin{array}{ccc}
z_{11} & z_{12} & z_{13} \\
z_{21} & z_{22} & z_{23} \\
z_{31} & z_{32} & z_{33}
\end{array}\right)=z_{11} z_{22} z_{33}-z_{11} z_{23}^{2}-z_{22} z_{13}^{2}
$$

Since the quadratic invariant $\operatorname{det} Z_{33}$ is positive, the equation (10) could represent either an ellipse (real or complex) or a single point in the $(\dot{\alpha}, \dot{\delta})$ plane. The linear invariant is always positive, thus the equation (10) represents a single point if and only if $\operatorname{det} Z=0$, while it represents a real ellipse if $\operatorname{det} Z<0$ and a complex ellipse if $\operatorname{det} Z>0$.

Then the condition to study for a real ellipse is

$$
z_{11} z_{22} z_{33}-z_{11} z_{23}^{2}-z_{22} z_{13}^{2}<0
$$

that is equivalent to

$$
z_{33}<\frac{z_{23}^{2}}{z_{22}}+\frac{z_{13}^{2}}{z_{11}}
$$

or explicitly

$$
\dot{\rho}^{2}+w_{1} \dot{\rho}+w_{4}-\frac{2 \mu_{E}}{\sqrt{S(\rho)}}<\left\langle\dot{P}_{O}, \widehat{R}_{\delta}\right\rangle^{2}+\frac{\left\langle\dot{P}_{O}, \widehat{R}_{\alpha}\right\rangle^{2}}{\cos ^{2} \delta} .
$$

Figure 5 shows a sketch of an admissible region in the plane $(\dot{\alpha}, \dot{\delta})$ : note that the ellipse has the axes parallel to the lines $\dot{\alpha}=0$ and $\dot{\delta}=0$ (in fact the polynomial $F$ does not contain the term $\dot{\alpha} \dot{\delta}$ ).

As observed for optical data we would like to to exclude orbits of "justlaunched" objects or too close objects. Then it is possible to fix a lower 


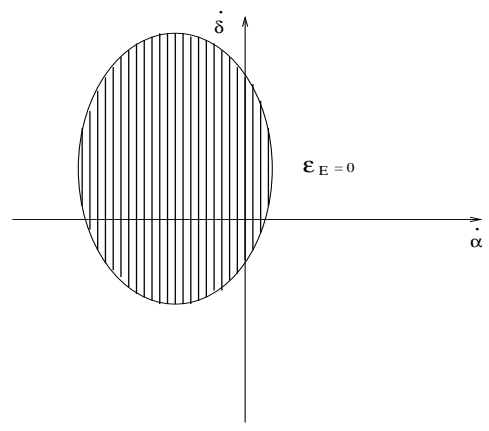

Figure 5: Fig. 5. Admissible region for a space debris $\mathcal{D}$ from radar data when condition (11) is satisfied: $\mathcal{E}_{E}=0$ is the curve of zero geocentric energy and it is an ellipse.

bound for the energy and the definition of admissible region could be modified:

$$
\mathcal{C}=\left\{(\dot{\alpha}, \dot{\delta}): \mathcal{E}_{E}^{\min }<\mathcal{E}_{E}<0\right\} .
$$

This new condition, if the curve $F(\dot{\alpha}, \dot{\delta})=0$ is a real ellipse, defines an elliptic corona in the $(\dot{\alpha}, \dot{\delta})$-plane as shown in Figure 6. In fact the curve $F(\dot{\alpha}, \dot{\delta})=\mathcal{E}_{E}^{\min }$ is a concentric ellipse (eventually it could be a point, the center of the ellipse, or a complex ellipse).

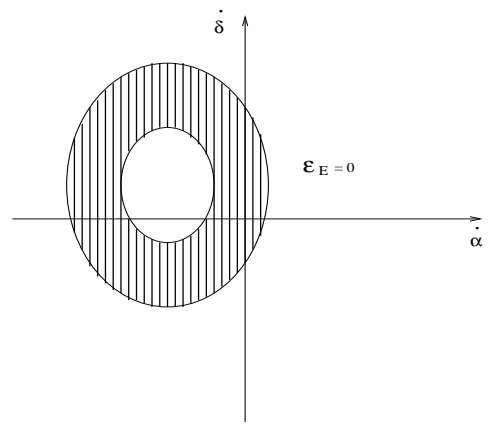

Figure 6: Admissible region for a space debris $\mathcal{D}$ from radar data when condition (11) is satisfied and taking into account the observation: $\mathcal{E}_{E}=0$ is the curve of zero geocentric energy and it is an ellipse.

\subsection{Sampling of the admissible region with a cobweb}

As explained in Subsection 2.1, if we have a a very short arc of observations we can compute the corresponding attributable (radar in this case) at some 
epoch, but we do not have enough information to obtain a least square solution.

If we assume that the object is a satellite of the Earth we can limit the uncertainty to the appropriate admissible region in the $(\dot{\alpha}, \dot{\delta})$, as illustrated before. In the case of optical observations we proposed to use the Delaunay triangulation as sampling of the admissible region. Now, since the region to sample is an ellipse, we suggest a different kind of sampling. The idea is to construct a cobweb (as described in [Tommei, 2006]), starting from a grid in the space of polar elliptic coordinates: this means to cover the admissible region $\mathcal{C}$ with the level curves of the geocentric energy and then, for each level curve, to select some points, corresponding to some fixed directions starting from the center of the ellipse. The points obtained are used as $\mathrm{VD}$ in a process of orbit determination. If we need to give a geometrical structure to these points we can triangulate the admissible region starting from the cobweb. This is possible simply working in the space of polar elliptic coordinates where the cobweb is a rectangular grid: we halve the cells composed by four nodes with a diagonal obtaining two triangles. Then joining the origin of the grid with the nodes of the first vertical line the triangulation is completed.

\section{Linkage and Identification}

When a debris object is observed for a short arc the information contained in such a data set are not enough to compute a full orbit, that is, a set of six orbital parameters. In such a case, the orbit determination must begin with the linkage of two TSAs (identification of two TSAs belonging to the same object). If we find an orbit it will be of very poor accuracy, so we have to look for another TSA to attribute to the previous orbit. We shall explain a procedure of orbit determination just tested on asteroids [Milani et al., 2005b] and we shall show how to apply it to debris objects. The procedure starts with a TSA, which can be composed by optical observations or by radar observations. First of all, the attributables (optical or radar) are computed by fitting the observations of the available short arcs. Let us suppose to have two attributables belonging to the same object: the steps leading to the orbit determination could be the following.

1. The admissible region of the first attributable is computed and sampled by a Delaunay triangulation (optical observations) or by the the cobweb (radar observations), providing a set of VD objects. 
2. The predictions for the time of the second arc, computed from the VD of the first, are compared with the second arc attributable.

3. For the VD, such that the attribution penalty (with the second attributable, see [Milani et al., 2005b]) is low, a preliminary orbit is computed. We recall that, given an attributable, the attribution penalty is defined as a measure of the likelihood that another attributable, computed from an independently detected TSA, actually belongs to the same object.

4. The above preliminary orbits are used as first guesses in constrained differential corrections [Milani et al., 2005a], providing, when there is convergence, LOV solutions fitting both very short arcs.

In this way the essentially 2-dimensional uncertainty (a thin region surrounding a copy of the admissible region) is reduced to an essentially 1dimensional uncertainty (a tubular neighbourhood of the LOV). If a third attributable is available we can reach a single nominal least squares solution with covariance, which could be used for additional attributions when more information is available.

\section{Observation of space debris}

In the current space debris measurement campaigns there are a number of examples where the method outlined in this paper can be of help. As mentioned earlier, beside the routine tracking performed by the SSN's, there are sporadic observational campaigns performed worldwide with the purpose of characterizing mainly the population of small debris objects. These campaign include optical campaigns, to characterize the environment in the GEO region for objects down to a few tens of $\mathrm{cm}$ (not included in the TLE Catalog), and radar observations campaigns, to measure the debris fluxes in the LEO region down to a size of a few $\mathrm{mm}$ (see [Schildknecht et al., 2005], [Barker et al., 2005], [Agapov et al., 2005], [Mehrholz et al., 2002], [Stansbery et al., 2005]).

The identification problem is apparent in the reports from the ESA 1$\mathrm{m}$ Telescope observations [Schildknecht et al., 2005]. The information obtained in the surveys made since 1999 are mainly statistical since no attempt was made to catalog the objects (the same is true for NASA CDT observations described in [Barker et al., 2005]). This means that some objects may have been observed multiple times. From a probabilistic analysis, in [Jehn et al., 2005] it is pointed out that the population of debris, brighter 
than visual magnitude 18.5, inferred from the ESA 1-m Telescope surveys, may indeed suffer from multiple observations. This might have lead to the over-estimation of this particular population by a factor of about 5 . An efficient algorithm for the identification could remove these ambiguities and allow a much better characterization of the GEO debris environment. Even more striking are the limitations that occur when objects in high elliptical orbits (such as the Geostationary Transfer Orbit, GTO) are observed. In these cases the very short observation arc of a few minutes does not allow to determine the full orbit and, of course, the usual assumption of a circular orbits (used for GEO observations) cannot be applied. Therefore, in order to determine elliptical orbits, long interval of observations with real time follow-up are necessary. This is a very time consuming scheduling that limits the number of objects actually determined in the surveys and that could greatly benefit from an efficient identification algorithm which could be based on the admissible region technique.

In the last decade a number of LEO debris observation campaigns have been performed using different radar sensors (e.g., Haystack, FGAN, Goldstone, Effelsberg) used in the so-called beam-park mode. In this operating mode, the radar beam is maintained in a fixed direction with respect to the Earth and all objects that pass through the beam are registered. From the echo of the radar signal, the size of the object and some of its orbital parameters can be determined. In LEO, the objects cross the radar field-of-view for a very short period of time. These radars usually works in pulse mode. A LEO object passes through the radar beam in only 3 or 4 pulses. Therefore, without follow-up measurements, usually only poor orbital data can be extracted and, typically, no information on eccentricity can be obtained. Again, an algorithm such as the one described here might help in optimizing the results of these radar campaigns.

It is also worth stressing that, although the SSN purpose is mainly to maintain of the TLE Catalog (and not to perform "blind" surveys looking for new objects), even this task is limited by procedural and observational constraints, where the identification difficulties play a significant role [Stansbery et al., 2005].

A final consideration is that, while until now the concept of a SSN was limited to United States and Russia, in the last years also other countries started to set up and design their own surveillance systems. In particular, ESA has financed a study for a European Space Surveillance System (ESSS) [Donath et al., 2005]. The ESSS, like the US SSN, should include two subsystems, one for LEO and another one for GEO observations. The LEO part should benefit from the experience of the French Space Surveillance System, 
called GRAVES, that is already operational [Michal et al., 2005].

\section{Conclusions and future work}

This work is a preliminary study on the possibility to realize a procedure of orbit determination of space debris starting from a data set which may be large, but still contains few observations per object. The idea is to extract information from all the available data in order to improve the knowledge of the space debris population.

We can summarize the conclusions obtained in the following items:

- we have defined and computed analytically the admissible region for a space debris object observed both by optical and radar sensors;

- we have shown how to generate a finite set of initial condition orbits to start an orbit determination procedure;

- we have explained a possible strategy to reduce a 2-dimensional uncertainty to a 1-dimensional uncertainty in order to perform an identification and thus a full orbit determination.

Since this theoretical study has showed the feasibility of the project, the future work will consist in the development of the software suitable to analyze real data. But it is worth stressing that this next step will require a massive effort in software writing and data analysis. It is going to be a large software suite and to be properly tested it will run on a large data set.

We note that the ESA Space Debris telescope (and the NASA ones) are currently operating since several years and no theory or procedure have yet been found to efficiently and effectively handle the problem of identification. The aim of our future work will be to provide a software able to overcome the present dangerous situation in which nearly all the GEO observations made worldwide (mainly by ESA and NASA) are currently useful only for statistical estimates, with large uncertainties, of the High Earth Orbit population.

Acknowledgements G.T. acknowledges the support by a Research Contract from ISTI-CNR (Pisa). The authors wish to thank M. Granvik for his comments.

\section{References}

[Agapov et al., 2005] Agapov, V., Biryukov, V., Kiladze, R., Molotov, I., Rumyantsev, V., Sochilina, A., Titenko, V.: Faint GEO Objects Search 
and Orbital Analysis. In: Proceedings of the Fourth European Conference on space debris, Darmstadt, Germany, ESA SP-587, 153-158, (2005)

[Barker et al., 2005] Barker, E., Jarvis, K., Africano, J., Jorgensen, K., Parr-Thumm, T., Matney, M., Stansbery, G.: The GEO environment as determined by the Cdt between 1998 and 2002. In: Proceedings of the Fourth European Conference on space debris, Darmstadt, Germany, ESA SP-587, 135-140, (2005)

[Donath et al., 2005] Donath, T., Schildknecht, T., Brousse, P., Laycock, J., Michal, T., Ameline, P., Leushacke L.: Proposal for a European Space Surveillance System. In: Proceedings of the Fourth European Conference on space debris, Darmstadt, Germany, ESA SP-587, 31-38, (2005)

[Michal et al., 2005] Michal, T., Eglizeaud, J.P., Bouchard, J.: GRAVES: the new French system for space surveillance. In: Proceedings of the Fourth European Conference on space debris, Darmstadt, Germany, ESA SP-587, 61-66, (2005)

[Jehn et al., 2005] Jehn, R., Ariafar, S., Schildknecht, T., Musci, R., Oswald, M.: Estimating the number of debris in the geostationary ring. Paper presented at the " $56^{\text {th }}$ International Astronautical Conference", Fukuoka, Japan, 2005

[Mehrholz et al., 2002] Mehrholz, D., Leushacke, L., Flury, W., Jehn, R., Klinkrad, H., Landgraf, M.: Detecting, tracking and imaging space debris, ESA Bulletin, 109, 128-134, (2002)

[Milani and Chesley, 2000] Milani, A., Chesley, S.R.: Virtual Impactors: Search and Destroy, Icarus, 145, 12-24, (2000)

[Milani et al., 2004] Milani, A., Gronchi, G.F., de' Michieli Vitturi, M., Knežević, Z.: Orbit determination with very short arcs. I admissible regions, Cel. Mech. Dyn. Ast., 90, 57-85, (2004)

[Milani et al., 2005a] Milani, A., Sansaturio, M.E., Tommei, G., Arratia, O., Chesley, S.R.: Multiple solutions for asteroid orbits: Computational procedure and applications, Astron. Astrophys., 431, 729-746, (2005)

[Milani et al., 2005b] Milani, A., Gronchi, G.F., Knežević, Z., Sansaturio, M.E., Arratia, O.: Orbit determination with very short arcs, Icarus, 179, $350-374,(2005)$ 
[Milani and Knežević, 2005] Milani, A., Knežević, Z.: From Astrometry to Celestial Mechanics: Orbit Determination with Very Short Arcs, Cel. Mech. Dyn. Ast., 92, 1-18, (2005)

[Rossi, 2005a] Rossi, A.: Population models of space debris. In: Milani, A.,Knežević, Z. (eds), Dynamics of Population of Planetary Systems, Proceedings of IAU Coll., 197, 427-438, CUP, (2005)

[Rossi, 2005b] Rossi, A.: The Earth orbiting space debris, Serb. Astron. J., $170,1-12,(2005)$

[Schildknecht et al., 2005] Schildknecht, T., Musci, R., Flury, W., Kuusela, J., de Leon, J., de Fatima Dominguez Palmero, J: Optical observations of space debris in high-altitude orbits. In: Proceedings of the Fourth European Conference on space debris, Darmstadt, Germany, ESA SP$587,113-118,(2005)$

[Stansbery et al., 2005] Stansbery, G., Foster, G.L. Jr.: Completeness of measurements of the orbital debris environment. In: Proceedings of the Fourth European Conference on space debris, Darmstadt, Germany, ESA SP-587, 95-100, (2005)

[Tommei, 2006] Tommei, G.: Impact Monitoring of Near-Earth Objects: theoretical and computational results, Ph.D. Thesis, University of Pisa, (2006) 